\title{
Fit für die Schule
}

\section{Was sollten Eltern wissen, um Schulproblemen vorzubeugen und Lernstörungen frühzeitig entgegenzuwirken? Eine Broschüre, die Sie im Wartezimmer auslegen können, gibt Antworten auf solche Fragen.}

$\mathrm{V}$ iele Eltern stehen den Schulproblemen ihrer Sprösslinge oft hilflos gegenüber. Die Patientenbroschüre „20 Fragen - 20 Antworten“ zum Thema „Fit für die Schule" beantwortet auf 28 Seiten die häufigsten Fragen, die Eltern von schulreifen Kindern oder Schülern beschäftigen. Typische Fragen: Was lässt sich, ohne gleich zu Medikamenten zu greifen, alles unternehmen, um den Kindern eine gute Lernumgebung zu schaffen? Oder: Was muss man bei der Ernährung beachten, um eine optimale Entwicklung zu ermöglichen? Wo findet man Rat, wenn man selbst nicht mehr weiter weiß?

Durch die Broschüre sollen die wichtigsten Probleme schon dargestellt und beantwortet werden, bevor es zum Arzt ins Sprechzimmer geht. So bleibt in der Sprechstunde mehr Zeit für gezieltere Fragen. Daneben ist sie für Eltern auch eine praktische Hilfe durch die Verweise auf weiterführende Webseiten und Adressen, bei denen sie Hilfsangebote abfragen können, oder eine kleine Nährwerttabelle für Lebensmittel, die Kinder für ein gesundes Aufwachsen benötigen.

Die Broschüre kann von allen Ärzten (immer im Paket von 100 Exemplaren) fürs Wartezimmer bestellt werden. Bestellungen per E-Mail an: vertrieb@springer.com oder per Fax an die Nr. 061 02/50 62 40, Stichwort „20 Fragen - Fit für die Schule“.

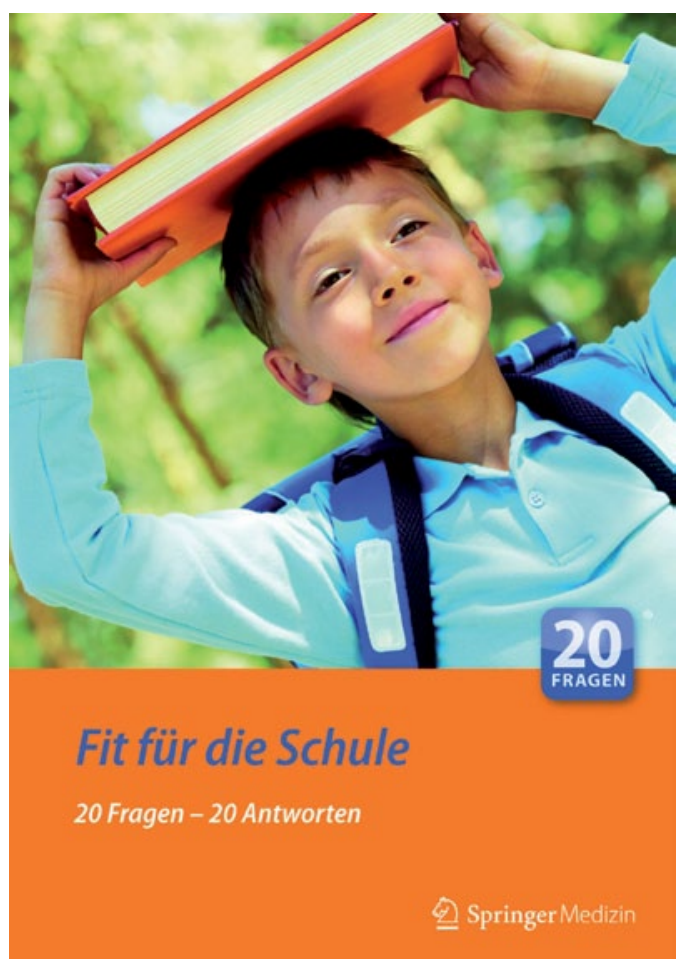

Kann im Wartezimmer ausgelegt werden: Die Broschüre „Fit für die Schule“.

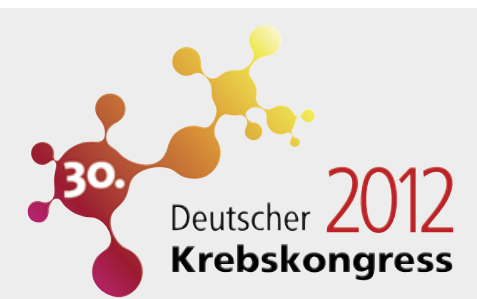

\section{Deutscher Krebskongress}

Berlin, 22. - 25. Februar 2012

Entwicklungen in der Krebsforschung aus erster Hand
Die steigende Zahl der Krebsneuerkrankungen ist alarmierend. Nur bessere Versorgungsstrukturen und optimierte Therapien können in dieser Situation weiterhelfen. Dazu braucht es eine exzellente Forschung und eine hohe Qualität in der Patientenversorgung. Um diesen Erfordernissen Nachdruck zu verleihen, wurde der kommende Deutsche Krebskongress unter das Motto "Qualität sichern - Forschung fo(e)rdern" gestellt. Denn Forschungsförderung sollte an wesentlichen Punkten durchaus auch politisch eingefordert werden.

\section{Besonderheiten des DKK 2012}

- Neben der Präsentation herausragender wissenschaftlicher Beiträge konzentriert sich das Programm auf gesundheitspolitische Themen. Im Rahmen der DKK-Veranstaltungen zum Nationalen Krebsplan werden Themen wie Krebsfrüherkennung, Qualitätssicherung, Arzneimittelbewertung und Patienteninformation diskutiert. Darüber hinaus wird es ein Symposium zur Qualität in der Patientenversorgung geben, das erstmalig in der Geschichte des DKK gemeinsam mit der renommierten amerikanischen Gesellschaft für klinische Onkologie ASCO durchgeführt wird. Symposien zur Forschungsförderung, zur Betreuung Langzeitüberlebender mit
Krebs und zu Krebs bei älteren Patienten runden das breite Spektrum der gesundheitspolitischen Veranstaltungen des DKK 2012 ab.

\section{Interdisziplinär und International}

- Der DKK zeichnet sich vor allem durch seine interdisziplinäre Ausrichtung aus - die klinische Onkologie ist dort ebenso vertreten wie die Radiotherapie, die onkologische Chirurgie, die Palliativmedizin und die onkologische Pflege. Um die Diskussion der vorgestellten Ergebnisse mit renommierten Kollegen aus dem In- und Ausland zu fördern, wurden außerdem neue Formate wie thematisch zusammenhängende Plenarsitzungen mit international besetzten „Key-Note-Lectures", „best-of"-Sitzungen und moderierte Diskussionsrunden ins Programm aufgenommen.

\author{
Weitere Informationen \\ - Das DKK-Vorprogramm kann ab sofort heruntergeladen werden: \\ www krebsgesellschaft.de/dkk_2012_programm. \\ - Unter folgendem Link können Sie einen Newsletter zum DKK 2012 \\ abonnieren: www.krebsgesellschaft.de/dkk_2012_presseverteiler
}

